\title{
3 Air toxics and other volatile organic compound emissions from unconventional oil and gas development
}

Arsineh Hecobian ${ }^{1 *}$, Andrea L. Clements ${ }^{1, \dagger}$, Kira B. Shonkwiler ${ }^{1, \$}$, Yong Zhou ${ }^{1}$, Landan P. MacDonald ${ }^{1, \#}$, Noel Hilliard ${ }^{1, \dagger}$, Bradley L. Wells ${ }^{1, \wedge}$, Bryan Bibeau ${ }^{2, \#}$, Jay M. Ham ${ }^{3}$, Jeffrey R. Pierce $^{1}$, Jeffrey L. Collett Jr. ${ }^{*}$

\section{Author Affiliations:}

$12{ }^{1}$ Department of Atmospheric Science, Colorado State University, Fort Collins, CO, U.S.A.

$13{ }^{2}$ Air Resource Specialists, Fort Collins, CO, U.S.A., 80526

14 '3 Department of Soil and Crop Sciences, Colorado State University, Fort Collins, CO, U.S.A., $15 \quad 80523$

16 Now at the U.S. Environmental Protection Agency National Exposure Research Laboratory,

17 Research Triangle Park, NC, U.S.A., 27709

18 Now at Colorado Department of Public Health and Environment, Denver, CO, U.S.A., 80246

19 \#Now at Dalhousie Medical School, Dalhousie University, Halifax, Nova Scotia, Canada,

20 B3H4R2

21 Now at MetStat, Fort Collins, CO, U.S.A., 80525

$22{ }^{\# N o w}$ at Thermo Fisher Scientific, Fort Collins, CO, U.S.A., 80526

$23{ }^{*}$ Corresponding authors

24 arsineh.hecobian@colostate.edu

25 Jeffrey.Collett@colostate.edu 
29 Tracer Ratio Method equation, where ER represents the emission rate, $\left[\mathrm{C}_{2} \mathrm{H}_{2}\right]$ is the 30 concentration of acetylene, [VOC] is the concentration of compound of interest, and [ $\mathrm{VOC}_{\mathrm{Bkg}}$ ] 31 and $\left[\mathrm{C}_{2} \mathrm{H}_{2 \mathrm{Bkg}}\right]$ denote background concentrations of the compound of interest and of acetylene, 32 respectively. 


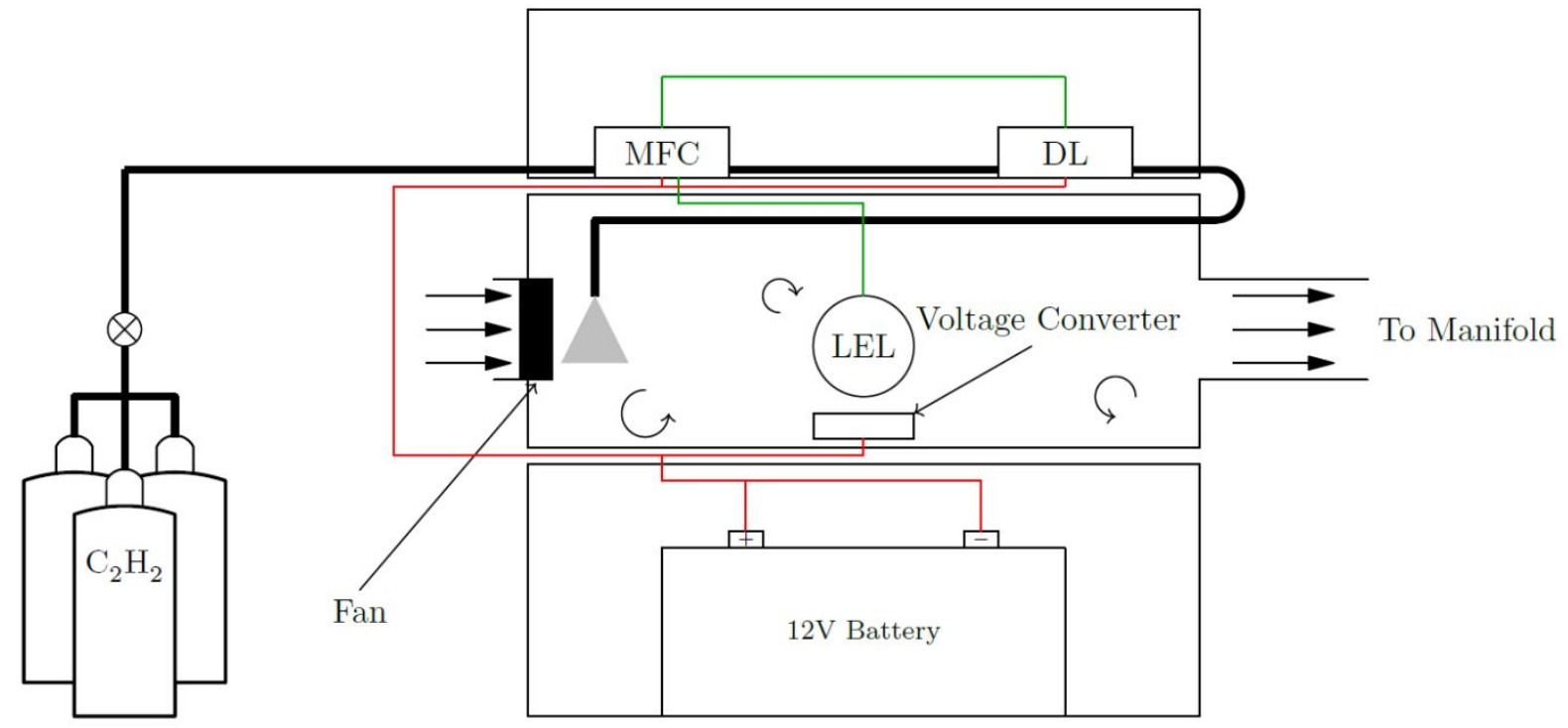

47

Figure S1. Diagram of the Tracer Release System (TRM). 
48

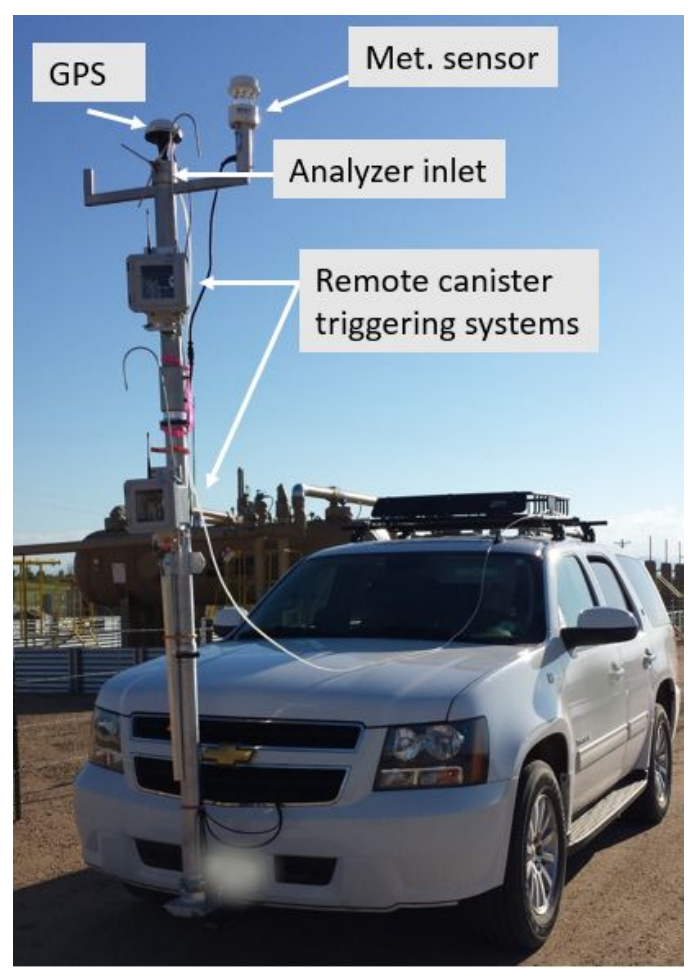

50 Fig. S2. Mobile Plume Tracker (MPT) with its external components. (Photo Credit: A.

51 Hecobian)

52 
53 Table S1. Description of the characteristics and benzene ERs for production sites.

\begin{tabular}{|c|c|c|c|c|c|c|c|c|}
\hline $\begin{array}{c}\text { Experiment } \\
\#\end{array}$ & $\begin{array}{c}\text { Monthly } \\
\text { gas } \\
(\text { Mscf })\end{array}$ & $\begin{array}{c}\text { Monthly } \\
\text { Condensate } \\
(\mathrm{bbl})\end{array}$ & $\begin{array}{c}\text { \# of } \\
\text { Wells }\end{array}$ & $\begin{array}{c}\text { \# of } \\
\text { Condensate } \\
\text { Tanks }\end{array}$ & $\begin{array}{c}\text { Stages of } \\
\text { Separation }\end{array}$ & Well Type & $\begin{array}{c}\text { Average } \\
\text { Benzene } \\
\text { ER (g/s) }\end{array}$ & $\begin{array}{c}\text { Stdev. } \\
\text { Benzene } \\
\text { ER (g/s) }\end{array}$ \\
\hline 1 & 613 & 18 & 9 & 3 & 1 & Vertical & 0.022 & 0.064 \\
\hline 2 & 3128 & 1047 & 4 & 5 & 3 & Horizontal & 0.0014 & 0.0011 \\
\hline 3 & 7197 & 707 & 11 & 0 & Bulk & Both & 0.030 & 0.048 \\
\hline 4 & 12378 & 1661 & 18 & 0 & Bulk & Horizontal & 0.0020 & 0.0027 \\
\hline 5 & 7715 & 1560 & 8 & 27 & 2 & Horizontal & 0.013 & 0.029 \\
\hline 6 & 251 & 25 & 6 & 3 & 2 & Both & 0.0010 & 0.00095 \\
\hline 7 & 1342 & 112 & 5 & 15 & 2 & Horizontal & 0.00081 & 0.0016 \\
\hline 8 & 21 & 1.1 & 2 & 2 & 2 & Vertical & 0.00074 & 0.00060 \\
\hline 9 & 5403 & 269 & 1 & 3 & 2 & Horizontal & 0.0023 & 0.0031 \\
\hline 10 & 189 & 189 & 2 & 4 & 2 & Vertical & 0.010 & 0.0066 \\
\hline 11 & 4527 & 3477 & 4 & 8 & 2 & Horizontal & 0.0064 & 0.0042 \\
\hline
\end{tabular}

\section{4}

55

56

57

58 
59 Table S2. Description of instruments used in the Mobile Plume Tracker.

60

61

62

\begin{tabular}{|l|l|l|}
\hline Instrument Type & Model & Manufacturer \\
\hline $\begin{array}{l}\text { Cavity Ringdown } \\
\text { Spectroscopy } \\
\text { (CRDS) methane and } \\
\text { acetylene analyzer }\end{array}$ & G2203 & Picarro \\
\hline Mobile kit & A0931 & Picarro \\
\hline GPS & A21 & Hemisphere GNSS \\
\hline $\begin{array}{l}\text { Wind speed and } \\
\text { direction }\end{array}$ & $102779-A 1-C 1-D 0$ & Climatronics \\
\hline
\end{tabular}

63 
64 Table S3. Study operations and the associated numbers of ER measurements by basin. 65

\begin{tabular}{|l|c|c|}
\hline Piceance basin & \multicolumn{2}{|c|}{} \\
\hline Type of operation & $\begin{array}{c}\text { Emission } \\
\text { measurements }\end{array}$ & Number of sites \\
\hline Drilling & 35 & 5 \\
\hline Fracking & 29 & 5 \\
\hline Flowback & 80 & 6 \\
\hline Denver-Julesburg basin & \\
\hline Type of operation & $\begin{array}{c}\text { Emission } \\
\text { measurements }\end{array}$ \\
\hline Fracking & 40 & 3 \\
\hline Flowback & 36 & 10 \\
\hline Production & 150 & 1 \\
\hline Liquids Load Out & 4 & Number of sites \\
\hline
\end{tabular}

66

67

68 
69 Table S4. Median ERs of VOCs measured for all sites and operations presented in this paper in 70 the Piceance and D-J basins.

\begin{tabular}{|c|c|c|c|c|c|c|c|}
\hline $\begin{array}{c}\text { VOC } \\
\text { (ppbv) }\end{array}$ & $\begin{array}{l}\text { Piceance } \\
\text { Drilling } \\
(\mathrm{g} / \mathrm{s})\end{array}$ & $\begin{array}{c}\text { Piceance } \\
\text { Fracking } \\
\quad(\mathrm{g} / \mathrm{s})\end{array}$ & $\begin{array}{c}\text { Piceance } \\
\text { Flowback } \\
(\mathrm{g} / \mathrm{s})\end{array}$ & $\begin{array}{l}\text { D-J } \\
\text { Fracking } \\
\quad(\mathrm{g} / \mathrm{s})\end{array}$ & $\begin{array}{l}\text { D-J } \\
\text { Flowback } \\
\quad(\mathrm{g} / \mathrm{s})\end{array}$ & $\begin{array}{l}\text { D-J } \\
\text { Production } \\
\quad(g / s)\end{array}$ & $\begin{array}{l}\text { D-J } \\
\text { Liquids } \\
\text { load out } \\
(\mathrm{g} / \mathrm{s})\end{array}$ \\
\hline ethane & 0.13 & 0.088 & 0.93 & 0.0026 & 1.10 & 0.10 & 6.6 \\
\hline ethene & 0.0030 & 0.0046 & 0.0030 & 0.0084 & 0.0011 & 0.0015 & 0.00066 \\
\hline propane & 0.12 & 0.013 & 0.37 & 0.00049 & 0.75 & 0.088 & 10.9 \\
\hline propene & 0.00032 & 0.0039 & 0.00091 & 0.0025 & 0.00037 & 0.00017 & 0.00017 \\
\hline i-butane & 0.0091 & 0.0036 & 0.15 & 0.00026 & 0.21 & 0.020 & 2.9 \\
\hline n-butane & 0.028 & 0.00032 & 0.12 & 0.00057 & 0.55 & 0.051 & 8.2 \\
\hline t-2-butene & 0.00024 & 0.00020 & 0.00013 & 0.00042 & 0.000044 & 0.000067 & 0.00045 \\
\hline 1-butene & 0.00017 & 0.00096 & 0.00010 & 0.00068 & 0.000069 & 0.000063 & 0.00034 \\
\hline c-2-butene & 0.00015 & 0.00031 & 0.00073 & 0.00060 & 0.0014 & 0.00017 & 0.013 \\
\hline cyclopentane & 0.0023 & 0.00067 & 0.0063 & 0.00026 & 0.023 & 0.00066 & 0.13 \\
\hline i-pentane & 0.0070 & 0.00041 & 0.11 & 0.00073 & 0.30 & 0.018 & 2.4 \\
\hline n-pentane & 0.0026 & 0.00027 & 0.081 & 0.00028 & 0.39 & 0.017 & 2.7 \\
\hline t-2-pentene & 0.00012 & 0.00013 & 0.00021 & 0.00041 & 0.000058 & 0.00022 & 0.035 \\
\hline 1-pentene & 0.00017 & 0.00031 & 0.00022 & 0.00069 & 0.000065 & 0.00011 & 0.00071 \\
\hline c-2-pentene & 0.000087 & 0.00021 & 0.0046 & 0.00040 & 0.088 & 0.0012 & 0.23 \\
\hline n-hexane & 0.0024 & 0.012 & 0.12 & 0.00059 & 0.29 & 0.0045 & 0.59 \\
\hline 2,3-dimethylpentane & 0.00044 & 0.0097 & 0.072 & 0.0033 & 0.10 & 0.00083 & 0.10 \\
\hline 2,4-dimethylpentane & 0.00011 & 0.00022 & 0.080 & 0.00090 & 0.144 & 0.0010 & 0.13 \\
\hline n-heptane & 0.0023 & 0.028 & 0.080 & 0.0013 & 0.19 & 0.0014 & 0.16 \\
\hline benzene & 0.0037 & 0.029 & 0.062 & 0.0022 & 0.069 & 0.0013 & 0.065 \\
\hline cyclohexane & 0.00053 & 0.023 & 0.11 & 0.00056 & 0.11 & 0.0014 & 0.20 \\
\hline 2-methylhexane & 0.00060 & 0.0072 & 0.022 & 0.0017 & 0.023 & 0.00034 & 0.034 \\
\hline 3-methylhexane & 0.00015 & 0.00078 & 0.093 & 0.0019 & 0.11 & 0.00098 & 0.13 \\
\hline 2,2,4-trimethylpentane & 0.00087 & 0.0088 & 0.020 & 0.0019 & 0.034 & 0.00069 & 0.053 \\
\hline 2,3,4-trimethylpentane & 0.00011 & 0.00037 & 0.0090 & 0.00084 & 0.25 & 0.00021 & 0.0090 \\
\hline methyl cyclohexane & 0.00079 & 0.085 & 0.50 & 0.0027 & 0.25 & 0.0021 & 0.25 \\
\hline toluene & 0.088 & 0.12 & 0.24 & 0.0056 & 0.21 & 0.0011 & 0.11 \\
\hline 2-methylheptane & 0.00059 & 0.034 & 0.10 & 0.0028 & 0.12 & 0.00072 & 0.053 \\
\hline 3-methylheptane & 0.00043 & 0.020 & 0.064 & 0.0017 & 0.063 & 0.00048 & 0.027 \\
\hline ethylbenzene & 0.00086 & 0.011 & 0.017 & 0.00084 & 0.0019 & 0.00022 & 0.0086 \\
\hline n-octane & 0.0031 & 0.096 & 0.23 & 0.0025 & 0.21 & 0.00091 & 0.085 \\
\hline $\mathrm{m}+\mathrm{p}$-xylenes & 0.0026 & 0.12 & 0.16 & 0.0040 & 0.24 & 0.0011 & 0.062 \\
\hline styrene & 0.0050 & 0.0047 & 0.0061 & 0.00095 & 0.0034 & 0.00043 & 0.011 \\
\hline o-xylene & 0.0015 & 0.021 & 0.038 & 0.0016 & 0.034 & 0.00052 & 0.013 \\
\hline n-nonane & 0.00054 & 0.097 & 0.17 & 0.0029 & 0.090 & 0.00068 & 0.028 \\
\hline i-propylbenzene & 0.0017 & 0.0047 & 0.010 & 0.0010 & 0.0066 & 0.00015 & 0.0011 \\
\hline n-propylbenzene & 0.00099 & 0.0031 & 0.0042 & 0.00063 & 0.0036 & 0.00012 & 0.00064 \\
\hline 2-ethyltoluene & 0.00071 & 0.020 & 0.018 & 0.0042 & 0.0039 & 0.0029 & 0.060 \\
\hline 3-ethyltoluene & 0.0014 & 0.015 & 0.027 & 0.0024 & 0.24 & 0.00022 & 0.0016 \\
\hline 4-ethyltoluene & 0.00094 & 0.0053 & 0.0092 & 0.0011 & 0.0035 & 0.00015 & 0.00080 \\
\hline 1,3-diethylbenzene & 0.00048 & 0.00091 & 0.0028 & 0.0040 & 0.0013 & 0.00030 & 0.0016 \\
\hline 1,4-diethylbenzene & 0.00034 & 0.00071 & 0.0080 & 0.0012 & 0.0044 & 0.00044 & 0.0011 \\
\hline 1,2,3-trimethylbenzene & 0.0034 & 0.015 & 0.016 & 0.0013 & 0.0064 & 0.00071 & 0.00091 \\
\hline 1,2,4-trimethylbenzene & 0.0025 & 0.035 & 0.056 & 0.0022 & 0.034 & 0.0024 & 0.0068 \\
\hline 1,3,5-trimethylbenzene & 0.00041 & 0.018 & 0.053 & 0.0014 & 0.027 & 0.00025 & 0.0069 \\
\hline n-decane & 0.0032 & 0.061 & 0.11 & 0.0051 & 0.042 & 0.00046 & 0.016 \\
\hline
\end{tabular}


72 Table S5. List of VOCs included in each category used in Fig. 1 of the paper.

73

\begin{tabular}{|l|l|}
\hline Categories & VOCs \\
\hline Light Alkanes & ethane, propane, i-butane, n-butane, i-pentane, n-pentane \\
\hline Heavy & 2,3-dimethylpentane, 2,4-dimethylpentane, 2,2,4-trimethylpentane, \\
Alkanes & $\begin{array}{l}\text { 2,3,4-trimethylpentane, n-hexane, 2-methylhexane, 3-methylhexane, } \\
\text { n-heptane, 2-methylheptane, 3-methylheptane, n-octane, n-nonane, n-decane }\end{array}$ \\
\hline Alkenes & $\begin{array}{l}\text { ethene, propene, t-2-butene, 1-butene, c-2-butene, t-2-pentene, 1-pentene, } \\
\text { c-2-pentene }\end{array}$ \\
\hline BTEX & benzene, toluene, ethylbenzene, o-xylene, m+p-xylenes \\
\hline Complex & $\begin{array}{l}\text { styrene, i-propylbenzene, n-propylbenzene, 1,2,3-trimethylbenzene, } \\
\text { Aromatics }\end{array}$ \\
& $\begin{array}{l}\text { 1,2,4-trimethylbenzene, 1,3,5-trimethylbenzene, 1,3-diethylbenzene, } \\
\text { 1,4-diethylbenzene, 2-ethyltoluene, 3-ethyltoluene, 4-ethyltoluene }\end{array}$ \\
\hline
\end{tabular}

74

75 